\title{
Factors associated with missed and delayed DTP3 vaccination in children aged 12 - 59 months in two communities in South Africa, 2012 - 2013
}

T N Mthiyane, ${ }^{1}$ BSc Hons, MSc; C Cohen, ${ }^{1,2}$ MB BCh, PhD; S A Norris, ${ }^{3}$ BSc Hons, PhD; S Walaza, ${ }^{1,2}$ MB BCh, MSc; S Tempia, ${ }^{2,4,5}$ DVM, MSc, PhD; A L Cohen, ${ }^{4,5,6}$ MD, MPH; A von Gottberg, ${ }^{2,7}$ MB BCh, DTM\&H, FC Path (SA) Micro, PhD; C von Mollendorf, ${ }^{1,2} \mathrm{MB} B C h, \mathrm{MSc}, \mathrm{PhD}$

${ }^{1}$ School of Public Health, Faculty of Health Sciences, University of the Witwatersrand, Johannesburg, South Africa

${ }^{2}$ Centre for Respiratory Diseases and Meningitis, National Institute for Communicable Diseases, National Health Laboratory Service, Johannesburg, South Africa

${ }^{3}$ MRC/Wits Developmental Pathways for Health Research Unit, Department of Paediatrics, Faculty of Health Sciences, University of the Witwatersrand, Johannesburg, South Africa

${ }^{4}$ Influenza Division, Centers for Disease Control and Prevention, Atlanta, Ga, USA

${ }^{5}$ Influenza Program, Centers for Disease Control and Prevention, Pretoria, South Africa

${ }^{6}$ Global Immunization Monitoring and Surveillance team, Expanded Programme on Immunization, Department of Immunization, Vaccines and Biologicals, World Health Organization, Geneva, Switzerland (current affiliation)

School of Pathology, Faculty of Health Sciences, University of the Witwatersrand, Johannesburg, South Africa

Corresponding author: C Cohen (cherylc@nicd.ac.za)

Background. Although immunisation services are available to all children in South Africa (SA), many children miss or have delays in receiving vaccines. There are limited data on factors associated with missed or delayed vaccination in children in this setting. Objectives. To assess vaccination coverage and factors associated with missed and delayed diphtheria-tetanus-pertussis vaccine third dose (DTP3) vaccination in children aged 12 - 59 months in two SA communities.

Methods. We used data from household-level healthcare utilisation surveys conducted in Soweto in 2012 and in Pietermaritzburg in 2013. Information on vaccination status was recorded from the Road to Health cards or vaccination history from clinics for children aged $<5$ years. Factors associated with missed or delayed DTP3 vaccination were assessed using unconditional logistic regression.

Results. Of a total of 847 eligible children aged $12-59$ months, 716 had available vaccination information. Overall DTP3 vaccination coverage was high for both sites: $90.6 \%$ in Pietermaritzburg and 93.9\% in Soweto. However, $32.6 \%$ and $25.2 \%$ of DTP3 vaccinations were delayed (received after 18 weeks of age) in Pietermaritzburg and Soweto, respectively. The median delay for DTP3 vaccinations was 4.7 weeks (interquartile range 1.7 - 23.0). Factors associated with delayed DTP3 vaccination included being born in 2010 (adjusted odds ratio (aOR) 3.0, 95\% confidence interval (CI) 1.4 - 6.3) or 2011 (aOR 2.7, 95\% CI 1.3 - 5.7) compared with being born in 2008, probably due to vaccine shortages; a low level of education of the primary caregiver, with children whose caregivers had completed secondary education having lower odds of delayed vaccination (aOR $0.5,95 \%$ CI 0.3 - 0.9) than children whose caregivers only had primary education; and maternal HIV status, with unknown status (aOR 3.5, 95\% CI 1.6 - 7.6) associated with higher odds of delay than positive status. Factors associated with missed DTP3 vaccination (not vaccinated by 12 months of age) included two or more children aged $<5$ years in a household (aOR $2.4,95 \%$ CI 1.2 - 4.9) compared with one child, and household monthly income <ZAR500 (aOR 3.4, 95\% CI 1.1 - 11.4) compared with $\geq$ ZAR2 000. Conclusions. Despite high overall DTP3 coverage observed in two communities, many vaccinations were delayed. Vulnerable groups identified in this study should be targeted with improved vaccination services to enhance uptake and timeliness of vaccination.

S Afr Med J 2019;109(8):562-569. DOI:10.7196/SAMJ.2019.v109i8.13244

Of 7.6 million children who died before their 5th birthday in 2010 , about two-thirds died of vaccine-preventable diseases. ${ }^{[1]}$ Sub-Saharan Africa had the highest under-5 mortality rate, with 92 deaths per 1000 live births in 2013. ${ }^{[2]}$ One possible factor contributing to the high mortality rate from vaccine-preventable diseases may be failure to complete all age-appropriate vaccine doses as specified by the World Health Organization (WHO) immunisation recommendations. ${ }^{[3]}$ Despite global improvements in vaccine coverage, coverage remains lowest on the African continent, with only $76 \%$ of children having received the third dose of diphtheria-tetanus-pertussis vaccine (DTP3) in 2015. ${ }^{[4]}$ Reported vaccination coverage in South Africa (SA) differs by province and district, as well as by the method used to determine coverage, i.e. local administrative data v. WHO/United Nations Children's Fund (UNICEF) vaccination coverage estimates. ${ }^{[5]}$ Based on administrative data, some districts reported vaccination coverage exceeding $100 \%$ for some vaccines, which implies inaccurate denominator data. ${ }^{[6]}$ According to the WHO/UNICEF estimates of national immunisation coverage, only $66 \%$ of children aged $12-23$ months in SA received DTP3 in 2016, which is below the recommended target of $90 \% .{ }^{[7]}$ DTP3 coverage at 12 months of age is considered to be the main indicator of immunisation programme performance, as it reflects the ability of the family to access and utilise immunisation services on multiple visits, and the functioning of the service. 
While immunisation programmes emphasise high coverage, delays in receipt of vaccinations are often not taken into consideration, ${ }^{[8]}$ and studies have shown that vaccination delays can occur even in countries with high vaccination coverage. ${ }^{[9]}$ Delayed vaccination leaves children susceptible to diseases and reduces herd immunity, which increases the spread of a disease in a population. It is therefore important to assess not only vaccine coverage but also timeliness of vaccination. Previous studies have recommended timely coverage as an indicator of immunisation programme performance, because high coverage alone cannot ensure adequate protection of children from infections. ${ }^{[10,11]}$ Delays increase with the number of vaccine doses; ${ }^{[12,13]}$ for example, DTP3 vaccination is more likely to be delayed than DTP1.

In SA, many children miss vaccinations or have delays in receiving them even though the National Department of Health $(\mathrm{NDoH})$ provides free vaccinations for all infants and children aged $<12$ years. A study that assessed timely delivery of immunisations to children during the first 2 years of life in a rural area of Eastern Cape Province found that half of diphtheria, tetanus, acellular pertussis, inactivated polio vaccine and Haemophilus influenzae type b (DTaP$\mathrm{IPV} / \mathrm{HiB})$ third immunisation doses were given $>4$ weeks late. ${ }^{[14]}$ In Western Cape Province, vaccination coverage rates $<90 \%$ and significant delays for most vaccines were observed. ${ }^{[13]}$ There are only a few studies available that looked at factors associated with missed and delayed vaccination in $\mathrm{SA}_{;} ;{ }^{[8,15]}$ these studies were also conducted before the introduction of new vaccines in the Expanded Programme on Immunisation (EPI). In 2009, three new vaccines, the pneumococcal conjugate vaccine (PCV), rotavirus vaccine and pentavalent vaccine with an acellular pertussis component, were all introduced into the EPI ${ }^{[16]}$ It is possible that these changes may have affected vaccine coverage or timely vaccination of children.

\section{Objectives}

To investigate vaccination coverage, and factors associated with missed and delayed vaccination, in children aged $<5$ years in two SA communities.

\section{Methods \\ Study setting and sampling}

This study used data from healthcare utilisation surveys (HUSs) conducted in areas surrounding two hospital-based severe acute respiratory infection surveillance sites, Chris Hani Baragwanath Hospital in Soweto (from September to December 2012) and Edendale Hospital in Pietermaritzburg (from October to December 2013). Soweto is an urban township outside Johannesburg with a population of $\sim 1.3$ million people in 2011. Pietermaritzburg is a periurban town with a population of 618536 in 2011. ${ }^{[17]}$

The study enrolled children aged $<5$ years from households that participated in the HUSs. For the Soweto HUS, households were selected for participation using a simple random sampling framework of geographical co-ordinates (latitude and longitude) within the boundaries of what were considered the residential areas of each site. ${ }^{[18]}$ The closest dwelling within $30 \mathrm{~m}$ of the randomly selected co-ordinates was approached for enrolment. ${ }^{[18]}$ For the Pietermaritzburg HUS, a complete list of households in the catchment area was available; households were then selected by simple random sampling. Field teams used hand-held global positioning system (GPS) devices to navigate to selected households.

\section{Data collection and outcomes}

Data were collected using standardised questionnaires through which members of the household were interviewed about demographic information, medical conditions and healthcare utilisation. The interviews were conducted in the preferred language of the household by community health workers. The information on vaccination status was recorded from Road to Health cards (RTHCs), and in Soweto also from clinic records when RTHCs were missing. Clinic visits for missing data were not done in Pietermaritzburg. In Soweto, nurses were able to obtain vaccination histories from clinic records if immunisation cards were not available, whereas in Pietermaritzburg vaccination histories were collected by community health workers who did not have access to clinic records. Children without an RTHC or vaccination history from clinic records were excluded. RTHCs or clinic records were also used to establish maternal HIV status. Selfreported maternal HIV status was used if status was not documented on the RTHC or clinic records.

Vaccination coverage was derived by dividing the number of children aged 12 - 59 months who received DTP3 vaccine by the number of children in that age group. A dose was considered given if it was recorded as such on the child's RTHC or in clinic records. The age of a child, calculated using date of birth and survey date, was used to determine whether or not a child was eligible to receive a specific vaccine dose as described in SA's EPI schedule for 2012 - 2013. Delayed vaccination coverage was calculated by dividing the number of children aged 12 - 59 months who received a vaccine dose $>1$ month from the due date by the total number of children who received that particular dose. We used a cut-off of 4 weeks because this is the minimum interval between doses that is recommended by the WHO. ${ }^{[19]}$ The age at vaccination was used to determine whether or not the vaccine dose was delayed and to estimate the length of delays (in weeks). A vaccine dose was considered missed if it was not received by 12 months of age. We also looked at the reasons given by caregivers for their children having missed or delayed any vaccinations, and challenges in accessing immunisation services.

\section{Statistical analysis}

All analyses were performed using Stata version 13 (StataCorp, USA). A $\chi^{2}$ test or Fisher's exact test was used to compare the characteristics of participants and vaccination coverage across study sites. Two separate logistic regression models were conducted to assess the association between independent variables and the vaccination outcomes (missed and delayed vaccination). All variables with a $p$-value $<0.20$ on univariate analysis were included in the multivariable model. Variables that were non-significant $(p>0.05)$ were removed one at a time using backward elimination, and the Wald test was used to confirm their significance in the model before removing them. A clustered sandwich estimator approach was used to account for clustering at the household level, as all children aged 12 - 59 months in the household were enrolled. Potential multicollinearity among independent variables was assessed prior to fitting the multivariable model. For example, age and birth year are highly correlated, so we maintained only one of these variables depending on its significance in the model.

\section{Ethical considerations}

The HUSs received approval from the University of the Witwatersrand (ref. no. M120367) and University of KwaZulu-Natal (ref. no. BE209/13) human research ethics committees, and the primary caregiver provided written consent for participation on behalf of the household. For this analysis, ethics approval was also obtained from the University of the Witwatersrand Human Research Ethics Committee (ref. no. M151164). 


\section{Results}

\section{Study participants}

Of a total of 2382 households that were randomly selected from a list of households in Pietermaritzburg, 144 (6.0\%) were no longer inhabited (Fig. 1). Of the remaining $2232(94.0 \%)$ that were visited, $134(6.0 \%)$ refused participation and $168(8.0 \%)$ did not have a primary caregiver present after three attempted visits for interview. A resulting 1936 (86.5\%) households with a total of 9750 members were surveyed in Pietermaritzburg. Among the surveyed households, 514 (26.5\%) had at least one child aged $<5$ years. In Soweto, of 1713 selected geographic co-ordinates, 191 (1.2\%) were no longer inhabited. Of the 1522 (88.8\%) households visited, 207 (13.6\%) refused participation and 342 (22.5\%) did not have a primary caregiver present after three attempted visits for interview. A resulting 973 (63.9\%) households with a total of 4364 members were interviewed in Soweto. Among the surveyed households, $248(25.5 \%)$ had at least one child aged $<5$ years. Data were collected on a total of 1061 children aged $<5$ years (705 from Pietermaritzburg and 356 from Soweto). Of these children, 847 were 12 - 59 months of age, $554(65.4 \%)$ from Pietermaritzburg and 293 (34.6\%) from Soweto. Of 847 enrolled children aged 12 - 59 months, only $716(84.5 \%)$ (279 (39.0\%) in Soweto and 437 (61.0\%) in Pietermaritzburg) had immunisation cards/vaccination histories.

\section{Characteristics of participants}

The study sites differed in respect of a number of characteristics (Table 1). In Soweto, households were significantly more likely than households in Pietermaritzburg to have a monthly income between ZAR500 and ZAR1 999 and primary caregivers who had completed secondary education. A higher proportion of children in Soweto lived in households made of bricks and with flush toilets. In Pietermaritzburg, households were more likely to report owning assets, such as cell phones (mobile phones), land or a bicycle, than households in Soweto.

\section{DTP3 vaccination coverage}

For the 716 children with available vaccination histories, DTP3 coverage was $>90 \%$ in both sites: $396 / 437(90.6 \%)$ in Pietermaritzburg and 262/279 (93.9\%) in Soweto. In Soweto, DTP3 coverage (Table 2) increased by age group and was highest in children aged 48 - 59 months (98.4\%), even though the increase was not significant. In

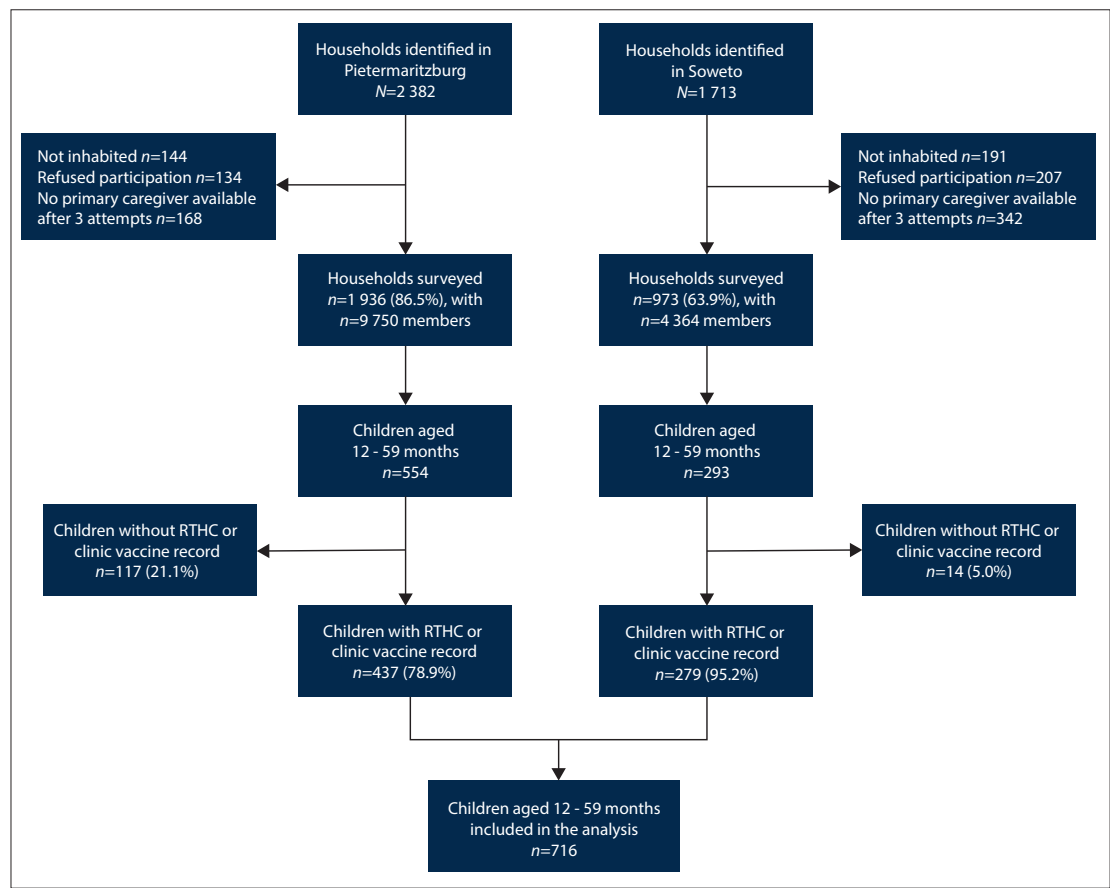

Fig. 1. Flow diagram of households and children from Pietermaritzburg and Soweto included in the healthcare utilisation surveys, South Africa, 2012 - 2013. (RTHC = Road to Health card.)

Pietermaritzburg there was no trend observed in coverage by age group. The median delay for DTP3 vaccination was more than a month (4.7 weeks, interquartile range $1.7-23.0$ ). DTP3 vaccinations were delayed by $32.6 \%$ and $25.2 \%$ in Pietermaritzburg and Soweto, respectively. Delayed DTP3 vaccination differed significantly by age group in both sites. The highest proportion of delayed DTP3 vaccination was observed among children aged 12-23 months in Soweto (36.7\%; $p=0.003)$ and among children aged 36 - 47 months in Pietermaritzburg (42.3\%; $p=0.001$ ).

\section{Factors associated with missed DTP3 vaccination}

Two factors were shown to be associated with children missing DTP3 vaccination at the two sites (Table 3). Children from households with two or more children aged $<5$ years (adjusted odds ratio $(\mathrm{aOR})$ 2.4, 95\% confidence interval (CI) 1.2 - 4.9) and children from households that had a monthly income <ZAR500 (aOR 3.4, 95\% CI 1.1 - 11.4) were more likely to miss DTP3 vaccinations than children from households with only one child aged $<5$ years or that had a monthly income of $\geq Z$ ZAR2 000 .

\section{Factors associated with delayed DTP3 vaccination}

Factors associated with delayed vaccination differed somewhat from those associated with missed vaccination (Table 4). Children whose mothers had completed secondary school education were less likely to have delayed vaccination than children whose mothers had only primary education (aOR 0.5, 95\% CI 0.3 - 0.9). Children born in 2010 (aOR 3.0, 95\% CI $1.4-6.3$ ) or 2011 (aOR 2.7, 95\% CI 1.3 - 5.7) were more likely to have delayed vaccination than children born in 2008. Unknown maternal HIV status was associated with increased odds of delayed vaccination (aOR 3.5, 95\% CI 1.6 - 7.6).

\section{Reasons given by caregivers for} missed or delayed vaccinations The two most common reasons given by caregivers for their children having missed or delayed receiving any vaccinations were that the clinic had been out of stock of the relevant vaccine $(147 / 253,58.1 \%)$ and that there was no one available to take the child to the clinic for immunisations (66/253, $26.1 \%)$. Of the children who missed or delayed any vaccinations due to vaccine stock-outs, 50 (34.0\%) and 58 (36.7\%) were born in 2010 and 2011, respectively.

\section{Challenges experienced by caregivers in accessing immunisation services for their children}

Only $6.8 \%(49 / 716)$ of caregivers reported challenges in accessing immunisation services for their children. The main challenges were unfriendly staff $(26 / 49$, $53.1 \%)$, restricted opening hours of clinics 


\begin{tabular}{|c|c|c|c|c|}
\hline Characteristic & Total & Pietermaritzburg & Soweto & $p$-value ${ }^{\star}$ \\
\hline Children, $N$ & 716 & 437 & 279 & \\
\hline \multicolumn{5}{|c|}{ Child's age (months), $n$ (\%) } \\
\hline $12-23$ & $199(27.8)$ & $112(25.6)$ & $87(31.2)$ & \\
\hline $24-35$ & $176(24.6)$ & $113(25.9)$ & $63(22.6)$ & \\
\hline $36-47$ & $171(23.9)$ & $103(23.5)$ & $68(24.4)$ & \\
\hline $48-59$ & $170(23.7)$ & $109(24.9)$ & $61(21.9)$ & 0.339 \\
\hline \multicolumn{5}{|c|}{ Child's sex, $n(\%)$} \\
\hline Male & $359(50.1)$ & $217(49.7)$ & $142(50.9)$ & \\
\hline Female & $357(49.9)$ & $220(50.3)$ & $137(49.1)$ & 0.746 \\
\hline Households, $N$ & 571 & 351 & 220 & \\
\hline \multicolumn{5}{|c|}{ Primary caregiver, $n(\%)$} \\
\hline Parent & $380(66.6)$ & $234(66.7)$ & $146(66.4)$ & \\
\hline Other & $191(33.4)$ & $117(33.3)$ & $76(33.6)$ & 0.940 \\
\hline \multicolumn{5}{|c|}{ Education of primary caregiver ${ }^{\dagger}, n(\%)$} \\
\hline Primary & $311(55.6)$ & $183(55.4)$ & $128(58.2)$ & \\
\hline Secondary & $82(14.9)$ & $10(3.0)$ & $72(32.7)$ & \\
\hline Tertiary & $157(28.5)$ & $137(41.5)$ & $20(9.1)$ & $<0.001$ \\
\hline \multicolumn{5}{|c|}{ Number of children aged $<5$ years, $n(\%)$} \\
\hline Only child & $360(63.0)$ & $227(64.7)$ & $133(60.4)$ & \\
\hline$\geq 2$ & $211(37.0)$ & $124(35.3)$ & $87(39.6)$ & 0.310 \\
\hline \multicolumn{5}{|c|}{ Maternal HIV ${ }^{\ddagger}, n(\%)$} \\
\hline Positive & $105(18.4)$ & $66(18.8)$ & $39(17.7)$ & \\
\hline Negative & $419(73.4)$ & $256(72.9)$ & $163(74.1)$ & \\
\hline Unknown & $47(8.2)$ & $29(9.4)$ & $18(8.2)$ & 0.946 \\
\hline \multicolumn{5}{|c|}{ Household income (ZAR),$n(\%)$} \\
\hline$<500$ & $35(8.5)$ & $12(5.2)$ & $23(12.7)$ & \\
\hline $500-1999$ & $234(56.5)$ & $128(54.9)$ & $106(58.6)$ & \\
\hline$\geq 2000$ & $145(35.0)$ & $93(39.9)$ & $52(28.7)$ & 0.005 \\
\hline \multicolumn{5}{|c|}{ Cell (mobile) phone, $n(\%)$} \\
\hline No & $25(4.4)$ & $6(1.7)$ & $19(78.6)$ & \\
\hline Yes & $546(95.6)$ & $345(98.3)$ & $201(91.4)$ & $<0.001$ \\
\hline \multicolumn{5}{|l|}{ Bicycle, $n(\%)$} \\
\hline No & $535(93.7)$ & $321(91.4)$ & $214(97.3)$ & \\
\hline Yes & $36(6.3)$ & $30(8.6)$ & $6(2.7)$ & 0.005 \\
\hline \multicolumn{5}{|c|}{ Own land, $n(\%)$} \\
\hline No & $474(83.0)$ & $263(74.9)$ & $211(95.9)$ & \\
\hline Yes & $97(17.0)$ & $88(25.1)$ & $9(4.1)$ & $<0.001$ \\
\hline \multicolumn{5}{|c|}{ Building material, $n$ (\%) } \\
\hline No bricks & $223(39.1)$ & $186(53.0)$ & $37(16.8)$ & \\
\hline Bricks & $348(60.9)$ & $165(47.0)$ & $183(83.2)$ & $<0.001$ \\
\hline \multicolumn{5}{|c|}{ Flush toilet, $n(\%)$} \\
\hline No & $323(56.6)$ & $273(77.8)$ & $50(22.7)$ & \\
\hline Yes & $248(43.4)$ & $78(22.2)$ & $170(77.3)$ & $<0.001$ \\
\hline \multicolumn{5}{|c|}{ Refrigerator, $n(\%)$} \\
\hline No & $52(9.1)$ & $26(7.4)$ & $26(11.8)$ & \\
\hline Yes & $519(90.9)$ & $325(92.6)$ & $195(88.2)$ & 0.075 \\
\hline $\begin{array}{l}\text { = Road to Healtt } \\
\text {-value compares } \\
\text { ortions calculated } \\
\text { rnal HIV status } \\
\text { ortions calculated }\end{array}$ & $\begin{array}{l}\text { nd Soweto. } \\
\text { ssing data (2 }\end{array}$ & & & \\
\hline
\end{tabular}

$(11 / 49,22.5 \%)$, long distances to travel to the clinic $(8 / 49,16.3 \%)$, being unable to get leave from work to take a child to the clinic $(3 / 49$, $6.1 \%)$ and transport costs to reach the clinic for immunisation services $(2 / 49,4.1 \%)$.

\section{Discussion}

In this study, we report DTP3 coverage and factors associated with delayed and missed vaccinations in children $12-59$ months of age using HUS data from two communities in SA. Our results 


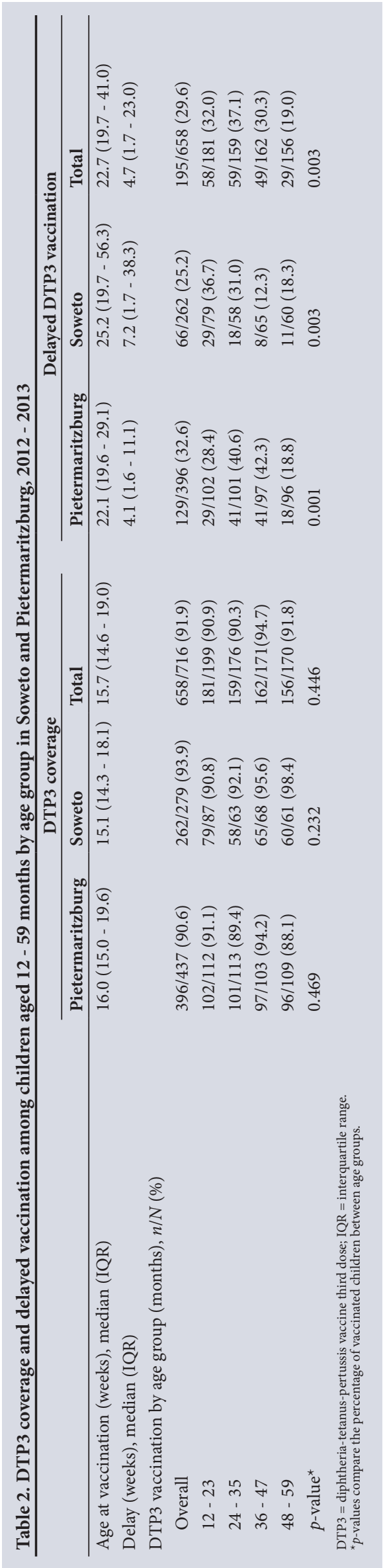

show significant variation in delayed vaccination between age groups, but no significant variation in vaccination coverage by age. Household characteristics that were associated with children missing vaccinations included low monthly income and having two or more children aged $<5$ years in a household. We found that being born in 2010 or 2011 and unknown maternal HIV status were associated with higher odds of delayed vaccination. Children whose mothers had completed secondary education were less likely to have delayed vaccination.

DTP3 vaccination coverage was high in both study sites. Different strategies used for vaccination history data collection at the different sites may have influenced coverage estimates, because we did not visit the clinics to verify vaccination histories in Pietermaritzburg. There are a number of estimates of vaccination coverage for $\mathrm{SA}$, including the $\mathrm{WHO}$ and $\mathrm{SA} \mathrm{NDoH}$ administrative estimates. ${ }^{[7]}$ These systems give differing results owing to use of different methodologies. ${ }^{[20]}$ WHO and UNICEF estimates for DTP3 in 2012 and 2013 were $65 \%$ and $73 \%$, respectively, while administrative DTP3 coverage was $89 \%$ and $91 \%$ in the same years. ${ }^{[7]}$ WHO estimates are derived through an annual country review of all available data, including administrative and surveybased coverage data. Administrative data estimates are calculated from health facility routine data in which the total number of children in the community forms part of the denominator and the number of vaccinations administered during a given period are included in the numerator. Our vaccination coverage estimates were similar to the administrative data, giving us some confidence in the validity of our findings. As we used a survey approach, it is possible that our results may be more accurate than other methods. Surveys produce estimates from a representative sample of households with children in a target age group and may include vaccine doses administered in private health facilities.

We observed a decrease in DTP3 coverage after new changes in the SA immunisation programme, i.e. the change to a combined vaccine (DTaP-IPV/Hib) and the introduction of new vaccines (rotavirus and PCV), all in 2009. Changing several different vaccines in the EPI at the same time may have affected the ability to timeously deliver vaccines to some degree, as clinics would have had to manage many different aspects of the new schedule including vaccine supply and the cold chain as well as staff training and other logistics. ${ }^{[21,22]}$ Stock-outs can affect vaccine delivery; in 2010, for example, the $\mathrm{NDoH}$ reported Pentaxim delivery issues that resulted in shortages of this vaccine in some health facilities in SA. ${ }^{[23]}$ Children born in 2010 or 2011 in our study were more likely to have delayed vaccinations, probably owing to vaccine delivery problems associated with changes to the EPI towards the end of 2009. A study in Burkina Faso found an association between year of birth and delayed vaccination that was related to changes in vaccination programme performance over time. ${ }^{[24]}$ Of the reasons given by caregivers in our study for their children having missed or delayed any of the vaccinations, vaccine stock-outs was the most common (58.1\%). In addition, a study in Malawi that assessed the predictors of uptake, timeliness and availability of new vaccines (rotavirus and PCV) found an association between vaccine stock-outs and delayed vaccination. ${ }^{[25]}$

Similar to previous studies, ${ }^{[26-28]}$ children in our study from households that had two or more young children were more likely to miss vaccinations. A possible reason for missing vaccinations in households with many children may be that the primary caregiver does not have the time or resources to take children to the clinic unless they are sick, and not just for routine vaccination visits. Children from households that had a lower income were more likely to be unvaccinated. These findings are consistent with previous studies in low- and middleincome countries. ${ }^{[27,29,30]}$ In Ethiopia, a study that assessed immunisation coverage and its determinants found that children in households with a family monthly income $>1000$ ETB (or 52 USD) were more likely to be fully vaccinated. ${ }^{[31]}$ Even though healthcare is free in SA for children aged $<5$ years, poor families may still have challenges with the transport costs involved in seeking regular healthcare.

Similar to previous studies in subSaharan Africa, we found that children whose primary caregiver had completed secondary education were less likely to have delayed vaccination than children whose mothers had only primary education. ${ }^{[2,32,33]}$ Caregivers who are educated are more likely to know the number of vaccine doses a child should receive and the age at which these doses should be received. ${ }^{[34]}$ They may also know the importance of timely vaccination. Contrary to a previous study in KwaZuluNatal Province that assessed the impact of maternal HIV on childhood vaccination 


\begin{tabular}{|c|c|c|c|}
\hline Factors & $n$ missed $/ N$ eligible & Unadjusted OR $(95 \% \mathrm{CI})^{*}$ & Adjusted OR $(95 \% \mathrm{CI})^{*}$ \\
\hline Site & & $p=0.933$ & \\
\hline Pietermaritzburg & $41 / 437$ & Reference & \\
\hline Soweto & $17 / 279$ & $0.9(0.6-1.5)$ & \\
\hline Age group (months) & & $p=0.449$ & \\
\hline $12-23$ & $18 / 199$ & Reference & \\
\hline $24-35$ & $17 / 176$ & $1.1(0.5-2.2)$ & \\
\hline $36-47$ & $9 / 171$ & $0.6(0.3-1.2)$ & \\
\hline $48-59$ & $14 / 170$ & $0.9(0.4-1.8)$ & \\
\hline Child's sex & & $p=0.767$ & \\
\hline Male & $28 / 359$ & Reference & \\
\hline Female & $30 / 357$ & $1.1(0.7-1.9)$ & \\
\hline Number of children in household aged $<5$ years & & $p=0.033$ & $p=0.018$ \\
\hline Only child & $21 / 360$ & Reference & Reference \\
\hline$\geq 2$ & $37 / 356$ & $1.9(1.1-3.3)$ & $2.4(1.2-4.9)$ \\
\hline Household income $(\mathrm{ZAR})^{\dagger}$ & & $p=0.190$ & $p=0.124$ \\
\hline$<500$ & $5 / 41$ & $2.6(0.8-6.9)$ & $3.4(1.1-11.4)$ \\
\hline $500-1999$ & $28 / 307$ & $1.8(0.9-3.4)$ & $1.8(0.8-4.0)$ \\
\hline$\geq 2000$ & $9 / 176$ & Reference & Reference \\
\hline Primary caregiver & & $p=0.088$ & \\
\hline Parent & $32 / 473$ & Reference & \\
\hline Other & $26 / 243$ & $1.7(0.9-2.9)$ & \\
\hline Education of caregiver ${ }^{*}$ & & $p=0.396$ & \\
\hline Primary & $34 / 387$ & Reference & \\
\hline Secondary & $5 / 104$ & $0.5(0.2-1.3)$ & \\
\hline Tertiary & $16 / 198$ & $0.9(0.5-1.8)$ & \\
\hline Maternal HIV & & $p=0.534$ & \\
\hline Positive & $8 / 134$ & Reference & \\
\hline Negative & $40 / 518$ & $1.3(0.6-3.1)$ & \\
\hline Unknown & $0 / 2$ & & \\
\hline
\end{tabular}

status and found that children of HIV-infected mothers had delayed vaccination, ${ }^{[17]}$ we found that unknown maternal HIV status was associated with delayed vaccination. In addition, maternal HIV prevalence estimated in this study was lower than the reported antenatal prevalence rates for the relevant provinces. In our study, maternal HIV prevalence was $18.5 \%$ in Pietermaritzburg (KwaZuluNatal) and $18.1 \%$ in Soweto (Gauteng), compared with $37.4 \%$ in KwaZulu-Natal and $29.9 \%$ in Gauteng. ${ }^{[35]}$ It is possible that known maternal HIV status could be associated with more frequent healthcare-seeking behaviour, which may affect the vaccination status of children. This was shown in a study from Zimbabwe that explored predictors of utilisation of maternal health services and women's health-seeking behaviour; it found that known positive or negative HIV status encouraged the use of maternal health services. ${ }^{[36]}$

\section{Study limitations and strengths}

Our study has several limitations. Firstly, household characteristics such as income and education of the primary caregiver were self-reported, which could have introduced misclassification bias. Secondly, of 654 participants with known maternal HIV status, only $91(13.9 \%)$ had documented HIV status on the RTHC or available from clinic records; it is therefore possible that we may have underestimated maternal HIV prevalence. Participants may have felt uncomfortable when asked to share sensitive information such as HIV status with community health workers as opposed to nurses, and may have therefore been more likely to withhold RTHCs with a documented HIV-positive status or missing vaccinations in Pietermaritzburg. Thirdly, households that agreed to participate in the HUSs could be different from those that refused participation, increasing the potential for selection bias; vaccination coverage in the community may have been overestimated if we assume that children from the households that refused as well as those that did not have RTHCs were unvaccinated. Fourthly, we may have slightly overestimated population coverage, as we excluded children without vaccination records. Lastly, our study used data from one urban site and one peri-urban site, and these results may therefore not be generalisable to all of SA, especially more rural populations.

Despite its limitations, the study also has several strengths. There are no studies to date in SA that have used HUS data to estimate vaccine coverage. Vaccination coverage estimates only included children with documented vaccination histories, so were not affected by recall bias. The household survey design, in which households were randomly selected, allowed us to collect information on the characteristics of a representative sample of households and 


\begin{tabular}{|c|c|c|c|}
\hline Factors & $n$ delayed/ $N$ eligible & Unadjusted OR $(95 \% \mathrm{CI})^{*}$ & Adjusted OR $(95 \% \mathrm{CI})^{\star}$ \\
\hline Site & & $p=0.091$ & \\
\hline Pietermaritzburg & $129 / 396$ & Reference & \\
\hline Soweto & $66 / 255$ & $0.7(0.5-1.1)$ & \\
\hline Birth year & & $p<0.001$ & $p<0.001$ \\
\hline 2008 & $15 / 79$ & Reference & Reference \\
\hline 2009 & $29 / 154$ & $1.0(0.5-2.1)$ & $0.9(0.4-2.0)$ \\
\hline 2010 & $66 / 165$ & $2.8(1.4-5.8)$ & $3.0(1.4-6.3)$ \\
\hline 2011 & $62 / 162$ & $2.6(1.3-5.4)$ & $2.7(1.3-5.7)$ \\
\hline 2012 & $23 / 91$ & $1.4(0.6-3.2)$ & $1.4(0.6-3.3)$ \\
\hline Child's sex & & $p=0.953$ & \\
\hline Male & $98 / 326$ & Reference & \\
\hline Female & $97 / 325$ & $1.0(0.7-1.4)$ & \\
\hline Number of children aged $<5$ years in household & & $p=0.876$ & \\
\hline Only child & $101 / 334$ & Reference & \\
\hline$\geq 2$ & $94 / 317$ & $1.0(0.7-1.4)$ & \\
\hline Household income $(\mathrm{ZAR})^{\dagger}$ & & $p=0.733$ & \\
\hline$<500$ & $10 / 35$ & $0.8(0.4-2.0)$ & \\
\hline $500-1999$ & $79 / 277$ & $0.8(0.5-1.3)$ & \\
\hline$\geq 2000$ & $53 / 164$ & Reference & \\
\hline Primary caregiver & & $p=0.730$ & \\
\hline Parent & $129 / 437$ & Reference & \\
\hline Other & $66 / 214$ & $1.1(0.7-1.5)$ & \\
\hline Education of primary caregiver ${ }^{*}$ & & $p=0.163$ & $p=0.078$ \\
\hline Primary & $111 / 348$ & Reference & Reference \\
\hline Secondary & $21 / 98$ & $0.6(0.3-1.0)$ & $0.5(0.3-0.9)$ \\
\hline Tertiary & $53 / 181$ & $0.9(0.6-1.3)$ & $0.8(0.5-1.2)$ \\
\hline Maternal HIV & & $p=0.010$ & $p=0.007$ \\
\hline Positive & $29 / 124$ & Reference & Reference \\
\hline Negative & $142 / 476$ & $1.4(0.9-2.2)$ & $1.6(1.0-2.7)$ \\
\hline Unknown & $24 / 51$ & $2.9(1.5-5.8)$ & $3.5(1.6-7.6)$ \\
\hline
\end{tabular}

individuals. Our sample included children of different age groups from two sites, so we were able to compare coverage estimates between these groups and sites. This study adds important data on vaccination coverage using a survey approach and on factors associated with missed and delayed vaccination.

\section{Conclusions}

Although DTP3 coverage was high overall in most age groups in our two communities, some of the vaccinations were delayed. This suggests that both coverage and timely vaccination should be considered as an indicator for immunisation programme performance. ${ }^{[9]}$ Children from households with low incomes, more than one child aged $<5$ years and primary caregivers with primary education should be targeted with improved vaccination services to increase uptake and timeliness. The health system needs to address issues of delivery to prevent unnecessary delays that occur due to vaccine stock-outs.

Declaration. This analysis was done by TNM as a partial requirement for her MSc (Epidemiology) degree.

Acknowledgements. We thank the interviewers and nurses for the collection of data and the data management team for data quality control.
Author contributions. CvM, CC, SW, ST, ALC and AvG contributed to the study concept and design. TNM, CvM, CC, SW, ST, ALC, AvG and SAN were responsible for acquiring, analysing or interpreting data. TNM drafted the manuscript. All authors critically revised the manuscript for important intellectual content.

Funding. This work was supported by the National Institute for Communicable Diseases of the National Health Laboratory Service and the US Centers for Disease Control and Prevention (co-operative agreement number: 5U51IP000155).

Conflicts of interest. None.

Disclaimer. The findings and conclusions in this article are those of the authors and do not necessarily represent the official position of the National Institute for Communicable Diseases or the Centers for Disease Control and Prevention.

1. Liu L, Johnson HL, Cousens S, et al. Global, regional, and national causes of child mortality An updated systematic analysis for 2010 with time trends since 2000. Lancet 2012;379(9832):2151 2161. https://doi.org/10.1016/S0140-6736(12)60560-1

2. World Health Organization and United Nations Children's Fund. Levels and trends in child mortality 2014. http://www.who.int/maternal_child_adolescent/documents/levels_trends_child_ mortality_2014/en/(accessed 23 May 2015).

3. World Health Organization. Recommended routine immunization for children (updated 21 May 2016). http://www.who.int/immunization/policy/Immunization_routine_table2.pdf?ua=1 (accessed 17 January 2017) 
4. Casey RM, Dumoland L, Danovaro-Holliday MC, et al. Global routine vaccination coverage, 2015. MMWR Morb Mortal Wkly Rep 2016;65:1270-1273. https://doi.org/10.15585/mmwr.mm6545a5

5. Bateman C. Vaccines: SA's immunisation programme debunked. S Afr Med J 2016;106(4):318-319. https://doi.org/10.7196/SAMJ.2016.v106i4.10765

6. Massyn N, Day C, Peer N, Padarath A, Barron P, English R. District Health Barometer 2013/14 Durban: Health Systems Trust, October 2014

7. World Health Organization and United Nations Children’s Fund. Progress towards global immunization goals (data as of 3 July 2017). http://www.who.int/immunization/monitoring_surveillance/data/za pdf (accessed 28 August 2017)

8. Fadnes LT, Jackson D, Engebretsen IM, et al. Vaccination coverage and timeliness in three South African areas: A prospective study. BMC Public Health 2011;11:404. https://doi.org/10.1186/1471 2458-11-404

9. Yadav K, Srivastava R, Kumar R, Chinnakal P, Rai SK, Krishnan A. Significant vaccination delay can occur even in a community with very high vaccination coverage: Evidence from Ballabgarh, India. J Trop Pediatr 2012;58(2):133-138. https://doi.org/10.1093/tropej/fmr059

10. Le Polain de Waroux O, Schellenberg JR, Manzi F, et al. Timeliness and completeness of vaccination and risk factors for low and late vaccine uptake in young children living in rural southern Tanzania. Int Health 2013;5(2):139-147. https://doi.org/10.1093/inthealth/iht006

11. Vasudevan L, Labrique AB, Mehra S, et al. Maternal determinants of timely vaccination coverage among infants in rural Bangladesh. Vaccine 2014;32(42):5514-5519. https://doi.org/10.1016/j vaccine 2014.06.092

12. Clark A, Sanderson C. Timing of children's vaccinations in 45 low-income and middle-income countries: An analysis of survey data. Lancet 2009;373(9674):1543-1549. https://doi.org/10.1016/ S0140-6736(09)60317-2

3. Corrigall J, Coetzee D, Cameron N. Is the Western Cape at risk of an outbreak of preventable childhoo diseases? Lessons from an evaluation of routine immunisation coverage. S Afr Med J 2008;98(1):41-45.

14. Le Roux K, Akin-Olugbade O, Katzen LS, et al. Immunisation coverage in the rural Eastern Cape - are we getting the basics of primary care right? Results from a longitudinal prospective cohort study. S Af Med J 2017;107(1):52-55. https://doi.org/10.7196/SAMJ.2017.v107i1.11242

15. Ndirangu J, Barnighausen T, Tanser F, Tint K, Newell ML. Levels of childhood vaccination coverage and the impact of maternal HIV status on child vaccination status in rural KwaZulu-Natal, South Africe Trop Med Int Health 2009:14(11):1383-1393. https//doi org/10 1111/j1365-31562009.02382

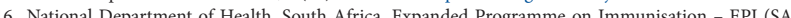
Revised Childhood Immunisation Schedule from April 2009. Pretoria: NDoH, 2015. http://www health.gov.za/index.php/component/phocadownload/category/165\# (accessed 20 April 2015).

17. Statistics South Africa. Census 2011. Pretoria: Stats SA, 2012. https://www.statssa.gov.za/ (accessed 5 May 2015).

18. Wong KK, Cohen AL, Norris SA, et al. Knowledge, attitudes, and practices about influenza illnes and vaccination: A cross-sectional survey in two South African communities. Influenza Other Resp Viruses 2016;10(5):421-428. https://doi.org/10.1111/irv.12388

19. World Health Organization. WHO recommendations for routine immunization - summary tables. (Last updated: 27 February 2015.) http://www.who.int/immunization/policy/immunization_tables/ en/ (accessed 28 July 2015)

20. Burton A, Monasch R, Lautenbach B, et al. WHO and UNICEF estimates of national infant immunization coverage: Methods and processes. Bull World Health Organ 2008;87(7):535-554 https://doi.org/10.2471/BLT.08.053819

21. Adegbola RA, Secka O, Lahai G, et al. Elimination of Haemophilus influenzae type b (Hib) disease from The Gambia after the introduction of routine immunisation with a Hib conjugate vaccine: A prospective study. Lancet 2005;366(9480):144-150. https:// 1 oi.org/10.1016/S0140-6736(05)66788-8
22. Griffiths UK, Korczak VS, Ayalew D, Yigzaw A. Incremental system costs of introducing combined DTwP-hepatitis B-Hib vaccine into national immunization services in Ethiopia. Vaccine 2009:27(9):1426-1432. https:///doi.org/10.1016/ivaccine.2008.12.037

23. Hu Y, Chen Y, Guo J, Tang X, Shen L. Completeness and timeliness of vaccination and determinants for low and late uptake among young children in eastern China. Human Vaccin Immunother 2014:10(5):1408-1415. https://doi.org/10.4161/hv.28054

24. Schoeps A, Ouedraogo N, Kagone M, Sie A, Muller O, Becher H. Socio-demographic determinants of adherence to BCG, Penta3, measles, and complete vaccination schedule in Burkina Faso. Vaccine 2014;32(1):90-102. https://doi.org/10.1016/.vaccine.2013.10.06

25. Mvula H, Heinsbroek E, Chihana M, et al. Predictors of uptake and timeliness of newly introduced pneumococcal and rotavirus vaccines, and of measles vaccine in rural Malawi : A population cohort study. PLoS One 2016;11(5):e0154997 https://doi.org/10.1371/journal.pone.0154997

26. Kawakatsu Y, Honda S. Individual, family- and community-level determinants of full vaccination coverage among children aged $12-23$ months in western Kenya. Vaccine 2012;14(30):7588-7593. https://doi.org/10.1016/j.vaccine.2012.10.037

27. Akmatov MK, Mikolajczyk RT. Timeliness of childhood vaccinations in 31 low and middle-income countries. J Epidemiol Community Health 2012;66(7):e14. https://doi.org/10.1136/jech.2010.124651

28. Danis K, Georgakopoulou T, Stavrou T, Laggas D, Panagiotopoulos T. Socioeconomic factors play a more important role in childhood vaccination coverage than parental perceptions: A cross-sectional more important role in childhood vaccination coverage than parental perceptions: A cross-s

29. Wiysonge CS, Uthman OA, Ndumbe PM, Hussey GD. Individual and contextual factors associated with low childhood immunisation coverage in sub-Saharan Africa: A multilevel analysis. PLoS One 2012;7(5):e37905. https://doi.org/10.1371/journal.pone.0037905

30. Clouston S, Kidman R, Palermo T. Social inequalities in vaccination uptake among children aged 0 59 months living in Madagascar: An analysis of demographic and health survey data from 2008 to 2009. Vaccine 2014;32(28):3533-3539. https://doi.org/10.1016/j.vaccine.2014.04.030

31. Legesse E, Dechasa W. An assessment of child immunisation coverage and its determinants in Sinana District, Southeast Ethiopia. BMC Pediatr 2015;15(1):31. https://doi.org/10.1186/s12887-015-0345-4

32. Gibson DG, Ochieng B, Kagucia EW, et al. Individual level determinants for not receiving immunization, receiving immunization with delay, and being severely underimmunized among rural western Kenyan children. Vaccine 2015;33(48):6778-6785. https://doi.org/10.1016/j.vaccine.2015.10.021

33. Animaw W, Taye W, Merdekios B, Tilahun M, Ayele G. Expanded program of immunization coverage and associated factors among children age 12-23 months in Arba Minch town and Zuria District, Southern Ethiopia, 2013. BMC Public Health 2014;14:464. https://doi. org/10.1186\%2F1471-2458-14-46

34. Bhuiya A, Bhuiya I, Chowdhury M. Factors affecting acceptance of immunization among children in rural Bangladesh. Health Policy Plan 1995;10(3):304-312. https://doi.org/10.1093/heapol/10.3.304

35. National Department of Health, South Africa. The 2013 National Antenatal Sentinel HIV Prevalence Survey South Africa. Pretoria: NDoH, 2015. http://www.health.gov.za/index.php/2014-03-17-09-0938/reports/category/176-reports-2015 (accessed 15 July 2016).

36. Maruva M, Gwavuya S, Marume M, Musarandega R, Madzingira N. Knowledge of HIV status at ANC and utilization of maternal health services in the 2010-11 Zimbabwe Demographic and Health Survey. DHS Working Papers No. 107 (Zimbabwe Working Papers No. 8). Rockville, Md: ICF International, 2014.

Accepted 8 October 2018. 